peripheral sympathetic nerves rescued the disrupted glucose metabolism phenotype of tumour-bearing mice. Furthermore, serum concentrations of leptin, which inhibits HO neurons, were reduced in tumour-bearing mice, suggesting a possible molecular explanation for the activation of $\mathrm{HO}$ neurons and the altered liver metabolism and sleep in these mice.

Overall, this study highlights the complex systemic effects a localized, non-metastatic tumour can have on its host. The authors state that similar effects were observed in mouse models of breast cancer using cells related to $67 \mathrm{NR}$ (4T1 and 4T07 cells), but the generalizability of this study, and relevance to humans, remains to be determined. If similar mechanisms exist in patients with cancer, then antagonism of $\mathrm{HO}$ neurons might be used therapeutically to improve metabolism and sleep.

Sarah Seton-Rogers

ORIGINAL ARTICLE Borniger, J. C., Walker II, W. H. et al. A role for hypocretin/orexin in metabolic and sleep abnormalities in a mouse model of non-metastatic breast cancer. Cell Metab. https:// doi.org/10.1016/j.cmet.2018.04.021 (2018)

The authors previously reported that a nutraceutical containing silibinin (which can impair STAT3 activation) had anti-tumour activity in two lung cancer patients with brain metastasis. They confirmed that silibinin inhibits pSTAT $^{+}$astrosphere formation in a STAT3-dependent manner, and showed that it reduced the metastatic lesion burden in mice after intracardiac or intracranial injection of $\mathrm{BrM}$ cells. Treatment of 18 patients with brain-metastatic lung cancer with silibinin led to a $75 \%$ overall response rate, with 3 complete responses. Comparison of these patients with others who did not receive silibinin indicated better control of brain metastasis with silibinin, as well as significantly improved overall survival (median 15.5 months versus 4.0 months).

There are several limitations of this clinical study, so further clinical trials are necessary, but inhibition of STAT3 is a promising avenue for targeting the brain microenvironment to treat metastasis.

Sarah Seton-Rogers

ORIGINAL ARTICLE Priego, N. et al. STAT3 labels a subpopulation of reactive astrocytes required for brain metastasis. Nat. Med. https:// doi.org/10.1038/s41591-018-0044-4 (2018)
TUMOUR MICROENVIRONMENT

\section{Metabolizing microbes in control}

Antitumour immune responses can be both promoted and inhibited by the intestinal microbiota. Although the liver is exposed to gut bacterial products and metabolites by way of the portal vein, it is unclear if the gut microbiota can influence the immunosurveillance of liver tumours. Ma et al. have now demonstrated in mice that the gut microbiota can metabolize bile acids to indirectly control liver tumour growth by modulating levels of hepatic natural killer T (NKT) cells.

To investigate how the gut microbiota might shape hepatic tumour development, the authors used a transgenic mouse model of primary hepatocellular cancer ( $\mathrm{HCC}$ ) and several models of liver metastasis. The commensal gut bacteria was altered in these mice by feeding them an antibiotic cocktail (ABX, comprised of vancomycin, neomycin and primaxin). ABX-treated mice with primary HCC developed smaller and fewer tumours whilst the subcutaneous implantation of EL4 lymphoma cells into $A B X$-treated mice did not affect primary tumour growth but did result in less spontaneous liver metastases. Similarly, intrasplenic injection of B16-F1 melanoma cells into ABX-treated mice led to reduced numbers of liver metastases. However, tail vein injection of B16-F1 cells induced an increase in lung metastases.

Collectively, these results suggest a liver-selective antitumour effect.

Concomitant with the inhibition of primary liver tumour growth and liver metastases upon depletion of the gut bacterial load was a specific accumulation of hepatic NKT cells expressing CXC-chemokine receptor 6 (CXCR6). This immune cell subset had an activated phenotype and following in vivo antigen stimulation produced higher levels of interferon- $\gamma($ IFN $\gamma)$. Depleting all three major $\mathrm{T}$ cell populations $\left(\mathrm{CD} 8^{+} \mathrm{T}\right.$ cells, $\mathrm{CD} 4$ $T$ cells and NKT cells) with antibodies in tumour-bearing mice or using tumour-bearing mice lacking hepatic NKT cells was sufficient to eliminate the antitumour response of $A B X$ treatment. Liver sinusoidal endothelial cells (LSECs), which represent an initial barrier for blood entering the liver from the gut, in $A B X$-treated mice exhibited increased surface expression of CXC-chemokine ligand 16 (CXCL16), the only ligand for CXCR6; a finding indicative of chemokine-mediated recruitment of NKT cells to the liver parenchyma.

The gut microbiota has been reported to modulate bile acid metabolism and composition.

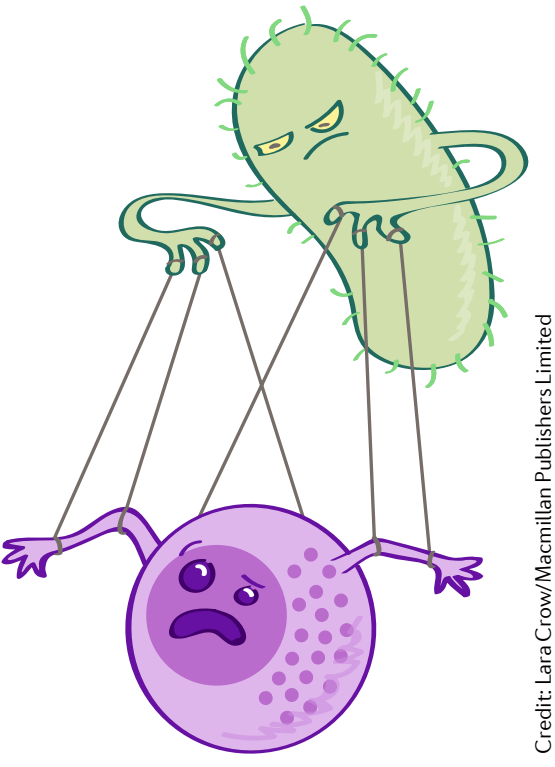

Hypothesizing that the conversion of primary bile acids into secondary bile acids by the gut commensal bacteria could be responsible for the regulation of NKT cell accumulation, the researchers observed that exposure of LSECs to primary bile acids increased Cxcl16 mRNA, whereas secondary bile acids had the opposite effect. This correlation was also observed in non-tumour liver tissues from patients with HCC.

To narrow down the bacterial species involved, Ma et al. found that individual antibiotic treatment with vancomycin alone caused an increase in hepatic NKT cells and a depletion of the Grampositive bacterial species Clostridium, known to perform the main enzymatic reaction in the generation of secondary bile acids. Importantly, colonization of vancomycin-treated mice with C. scindens or feeding secondary bile acids to vancomycin-treated mice resulted in a decrease in hepatic NKT cells and more liver metastases.

Although hepatic NKT cells are much less prevalent in humans, mucosal-associated invariant T (MAIT) cells expressing CXCR6 are commonly found in the human liver, suggesting bile acid signals mediated by the gut microbiota to alter immune cell function and promote liver cancer could be a universal mechanism.

Anna Dart

ORIGINAL ARTICLE Ma, C. et al. Gut microbiome-mediated bile acid metabolism regulates liver cancer via NKT cells. Science $\mathbf{3 6 0}$ eaan5931 (2018)

FURTHER READING Roy, S. \& Trinchieri, G. Microbiota: a key orchestrator of cancer therapy. Nat. Rev. Cancer 17, 271-285 (2017) 